\title{
Suppression of lung cancer with siRNA targeting PTTG
}

\author{
SHAM S. KAKAR and MOHAMMAD T. MALIK \\ Department of Medicine, James Graham Brown Cancer Center, University of Louisville, Louisville, KY 40202, USA
}

Received January 27, 2006; Accepted March 20, 2006

\begin{abstract}
Pituitary transforming gene (PTTG) is frequently expressed at high levels in malignant tumors. We report high levels of expression of PTTG in various lung tumors and tumor-derived cell lines. For a better understanding of its role in maintaining the cancer phenotype, we used RNA interference (RNAi) directed against PTTG. Transfection of H1299 cells with PTTG siRNA duplex (5'-UGG GAG AUC UCA AGU UUC A-3') to a final concentration of $100 \mathrm{nmol} / \mathrm{l}$ resulted in almost complete depletion of PTTG mRNA within 24 and $48 \mathrm{~h}$ when compared to expression in untransfected cells or cells transfected with control siRNA. Western blot analysis showed nearly a $60 \%$ reduction in PTTG protein within 48 h of transfection. In phenotype analysis, we investigated the effect of PTTG siRNA on colony formation on soft agar, and tumor development in nude mice. Transfection of H1299 cells with PTTG siRNA duplex significantly reduced colony formation compared to untransfected cells or cells transfected with control siRNA. Mice injected with H1299 cells transfected with PTTG siRNA followed by injection of siRNA developed no tumors within two weeks of injection, but developed small tumors $(67.85 \pm 45.87 \mathrm{mg})$ within 4 weeks of injection, whereas untransfected cells, or cells transfected with control siRNA developed large tumors $(232.12 \pm 102.78$ and $231.57 \pm 83.76 \mathrm{mg}$ respectively). Suppression of PTTG as well as Ki67, bFGF and CD34 was observed in H1299 tumors treated with PTTG siRNA compared to untreated and control-treated siRNA in nude mice. In conclusion, decreasing PTTG expression through PTTG siRNA inhibits tumor growth both in vitro and in vivo. Future studies are needed to test whether PTTG expression can be efficiently depleted by siRNA expressed from a DNAbased expression vector combined with a specific-promoter, such that RNAi can specifically target PTTG in cancer cells without affecting normal cells.
\end{abstract}

Correspondence to: Dr Sham S. Kakar, Department of Medicine, 580 South Preston Street, Baxter II, Room 321E, Louisville, KY 40202, USA

E-mail: sskaka01@louisville.edu

Key words: pituitary transforming gene, securin, small interfering RNA, lung cancer, tumor growth

\section{Introduction}

Lung cancer continues to be the leading cause of cancer related deaths in the United States and worldwide. There are expected to be 172,570 new cases of lung cancer with 163,510 cancer deaths in 2005 (1). This is in stark contrast to the combined United States mortality from colon, breast and prostate cancer, which together approximate 127,510. Lung cancer develops slowly over many years due to the sequential accumulation of gene alterations in susceptible cells (2). The two major types of lung cancer are non-small cell lung carcinoma (NSCLC) and small cell lung carcinomas (SCLC). NSCLC accounts for $75-85 \%$ of cases, and is further classified as adenocarcinoma (40\%) including bronchoalveolar carcinoma, sequamous cell carcinoma (30-35\%), and large cell carcinoma (5-15\%) (3). More than three quarters of all cases of NSCLC present with locally advanced disease or widespread metastases (stage IIIB and IV). Chemotherapy is the mainstay of treatment; however, overall survival is poor, and has not improved significantly over two decades. To improve the diagnosis and treatment of lung cancer, we need to find novel approaches that exceed those currently available.

Pituitary tumor transforming gene (PTTG), also known as securin, is a potent oncogene that was initially cloned from a rat pituitary tumor (4). We cloned a PTTG homolog from human testis and ovarian tumors $(5,6)$. Human PTTG encodes a protein of 202 amino acids, and is $85 \%$ identical with rat PTTG. PTTG has been localized to human chromosome 5q35.1 (7), a locus associated with recurrent lung cancer (8). Numerous studies have demonstrated that human PTTG displays a distinct pattern of expression. In normal human tissues, PTTG expression is very restricted, with high levels in the testis, and low levels in the thymus, colon, and small intestine. In contrast, PTTG is highly expressed in a variety of human primary tumors as well as tumor cell lines, including carcinoma of the ovary, testis, kidney, colon, thyroid, pituitary, liver, adrenal, and breast, and melanoma, leukemia, and lymphoma $(5,6,9-18)$, suggesting that PTTG may be an important gene involved in human tumorigenesis. Furthermore, the expression levels of PTTG correlate positively with increased tumor invasiveness in hormone-producing human pituitary tumors (14) and the degree of malignancy, pathogenesis and/or progression of colorectal, thyroid and breast tumors $(12,13,15)$. Recently, PTTG was identified as one of the signature genes among 8 associated with tumor metastasis that are up-regulated in human primary solid tumors (17). A relationship between the 
survival rate and the level of expression of PTTG in esophageal cancer has also recently been reported (18).

Overexpression of PTTG in mouse fibroblast (NIH 3T3) and human fetal kidney (HEK293) cells induces cellular transformation and promotes tumor formation in nude mice $(4,5,19)$. Overexpression of PTTG increases the expression and secretion of basic growth factor (bFGF) (19-21), VEGF $(19,22)$, and IL-8 (19), and plays a role in tumor angiogenesis and metastasis (20-22), implying that PTTG may be a fundamental oncogene and potential target for cancer diagnosis and therapy. To explore the role of PTTG in tumor growth and angiogenesis, we utilized a new technology, siRNA, to down-regulate the gene of interest. To avoid non-specific gene silencing induced by longer double-stranded RNA, small interfering RNA (siRNA), a duplex of 19 nucleotide, was used as a mediator to degrade target mRNA (23). Once inside the cell, siRNA is incorporated into an RNA-induced silence complex (RISC), a protein-RNA complex resulting in unwinding and strand separation of RNA duplex. The antisense RNA then guides the activated RISC to anneal and cleave the target RNA (24-27). In addition, several in vitro studies have demonstrated that siRNA can effectively reduce tumor ontogenesis (28-30).

In the present study, we utilized PTTG siRNA and found down-regulation PTTG mRNA and protein, and reduction in tumor growth in vitro and in vivo.

\section{Materials and methods}

Preparation of siRNA. A smart pool of four siRNA sequences derived from the coding sequence of PTTG and individual duplex and control siRNA were designed and purchased from Dharmacon (Lafayette, Co.). The siRNAs include PTTG siRNA duplex no. 1 (bases 581-599) 5'-GAC CCU GGA UGU UGA AUU G-3' (sense), duplex no. 2 (bases 64-82) 5'-GCA CCC GUG UGG UUG CUA A-3' (sense), duplex no. 3 (128-146) 5'-UGG GAG AUC UCA AGU UUC A-3' (sense), and duplex no. 4 (bases 177-195) 5'-CCA CCA GCC UUA CCU AAA G-3' (sense); and control siRNA 5'-NAU GUA UUG GCC UGU AUU A-3', where $\mathrm{N}$ is any nucleotide in the intended mRNA sequence. Each freeze-dried siRNA duplex was reconstituted with RNase-free water to prepare a $20 \mu \mathrm{M}$ stock solution. The target sequence of each siRNA and its location in PTTG cDNA is shown in Fig. 1.

Cell culture and transfection of cells with siRNA. Human non-small cell lung carcinoma cell line (H1299) was purchased from ATCC (American Type Culture Collection, Rockville, MD) and cultured according to the suppliers' instructions. For transfection, H1299 cells were plated in 6-well plates at a density of $2 \times 10^{5}$ cells/well. After $24 \mathrm{~h}$, cells were transfected with siRNA to a final concentration of $100 \mathrm{nmol} / \mathrm{l}$ using Mirus (Dharmacon) transfection reagent according to the manufacturer's instructions.

Northern blot analysis. A total of $2 \times 10^{5}$ cells/well were plated in 6-well plates and allowed to adhere for $24 \mathrm{~h}$. Cells were transfected with siRNA as described above. After 24-48 h of transfection, cells were washed with PBS buffer. Total RNA from tissues and cells was prepared using TRIzol reagent (Invitrogen, Carlsbad, CA) as described previously (5). The
RNA pellets were resuspended in RNase-free water, and the contaminating DNA was removed from the preparation with DNaseI. The yield of RNA was measured using a spectrophotometer. Twenty $\mu \mathrm{g}$ of total RNA was separated on a $1 \%$ agarose/formaldehyde gel, and was transferred to a nylon membrane (Amersham Biosciences), and crosslinked as described previously (5). The membrane was prehybridized for $30 \mathrm{~min}$ at $65^{\circ} \mathrm{C}$ in ExpressHyb buffer (Clontech Laboratories Inc., Palo Alto, CA). PTTG cDNA or GAPDH cDNA was labeled with $\left[\alpha_{-}{ }^{32} \mathrm{P}\right] \mathrm{dCTP}$ using a random hexamer primer labeling kit (Promega Co., Madison, WI). Hybridization was initially performed with labeled PTTG cDNA at $65^{\circ} \mathrm{C}$ for $1 \mathrm{~h}$. The membrane was washed three times with $2 \mathrm{X} \mathrm{SSC} / 0.1 \%$ SDS for $15 \mathrm{~min}$ each and then with $0.2 \mathrm{X} \mathrm{SSC/0.1 \%} \mathrm{SDS} \mathrm{at}$ $55^{\circ} \mathrm{C}$ for $30 \mathrm{~min}$ and exposed to X-ray film. The membrane was then stripped and rehybridized with labeled GAPDH cDNA.

Western blot analysis. To determine the level of PTTG protein, $2 \times 10^{5}$ cells were plated in 6-well plates and transfected as described above. After transfection, cells were washed with PBS and lysed in chilled lysis buffer [50 mM Tris- $\mathrm{HCl}$ (pH 7.5), $150 \mathrm{mM} \mathrm{NaCl}, 1 \% \mathrm{NP}-40,1 \mathrm{mM} \mathrm{Na}_{3} \mathrm{VO}_{4}$, and $1 \mathrm{mM}$ $\mathrm{NaF}$ ] supplemented with Complete Mini Protease Inhibitor tablets (Roche Molecular Biochemical, Indianapolis, IN). Equal amounts of protein extracts $(40 \mu \mathrm{g})$ were resolved on a $12 \%$ SDS-PAGE gel, and transferred onto a nitrocellulose membrane (Amersham Biosciences, Piscataway, NJ). Blots were probed with PTTG antiserum at a dilution of 1:1,500 as described previously (13). Immunoreactive proteins were visualized using the Enhanced Chemiluminescent Detection system (ECL) (Amersham Biosciences) according to instructions provided. The membrane was stripped and reprobed with $B$-actin antibody (Sigma Chemical Co., St. Louis, MO).

Soft agar colony formation (anchorage-independent cell growth assay). Anchorage-independent cell growth was determined by analyzing the formation of colonies on soft agar. Briefly, H1299 cells were transfected with PTTG siRNA or control siRNA for $48 \mathrm{~h}$ as described above. Cells $\left(10^{4}\right)$ from each sample were resuspended in $0.3 \%$ agar in DMEM containing $10 \%$ fetal bovine serum, and plated on solidified agar $(0.7 \%)$ in 35-mm dishes. After 14 days of culture, the colonies formed were counted and photographed as described previously (5).

Tumor growth in nude mice. The effect of siRNA on tumor growth was studied on 5- to 6-week-old nu/nu female mice (Charles River Laboratory, Wilmington, MA). Mice were divided into three groups, with five mice per group. H1299 cells were plated into T-75 flasks. After $24 \mathrm{~h}$, cells were transfected with PTTG siRNA duplex no. 3 or control siRNA to a final concentration of $100 \mathrm{nmol} / \mathrm{l}$. After $48 \mathrm{~h}$ of transfection, cells were washed with PBS, treated with trypsin, washed with PBS and then counted using a hemocytometer. Aliquots of $1 \times 10^{6}$ untransfected cells, cells transfected with PTTG siRNA or control siRNA were resuspended into $100 \mu 1$ PBS. Cells were injected s.c. into both flanks of mice. The mice were kept in a pathogen-free environment and examined every three days. Tumor diameter was measured at regular intervals with a digital caliper, and tumor volume in $\mathrm{mm}^{3}$ was 


\begin{tabular}{|c|c|c|}
\hline-20 & $\begin{array}{l}\text { AAGACCTGCAATAATCCAGAATGGCTACTCTGATCTATGT } \\
\text { START }\end{array}$ & 40 \\
\hline 41 & TGATAAGGAAAATGGAGAACCAGGCACCCGTGTGGTTGCT & 80 \\
\hline 81 & AAGGATGGGCTGAAGCTGGGGTCTGGACCTI & 120 \\
\hline 121 & CCTTAGATGGGAGATCTCAAGTTTCAACACCACGTTTTGG & 160 \\
\hline 161 & 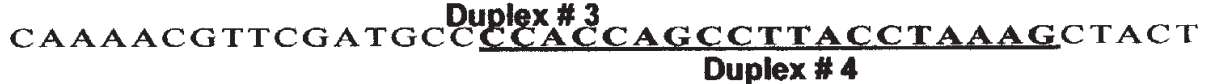 & 00 \\
\hline 201 & AGAAAGGCTTTGGGAACTGTCAACAC & 240 \\
\hline 241 & СTGTAAAGACCAAGGGACCCCTCAAACAAAAACAGCCAAG & 80 \\
\hline 281 & СTTTTCTGCCAAAAAGATGACTGAGAAGACTGTTAAAGCA & 20 \\
\hline 321 & AAAAGCTCTGTTCCTGCCTCAGATGATGCCTATCCAGAAA & 60 \\
\hline 361 & TAGAAAAATTCTTTCCCTTCAATCCTCTAGACTTTGAGAG & 00 \\
\hline 401 & TTTTGACCTGCCTGAAGAGCACCAGATTGCGCACCTCCCC & 40 \\
\hline 441 & TTGAGTGGAGTGCCTCTCATGATCCTTGACGAGGAGAGAG & 80 \\
\hline 481 & AGCTTGAAAAGCTGTTTCAGCTGGGCCCCCCTTCACCTGT & 20 \\
\hline 521 & GAAGATGCCCTCTCCACCATGGGAATCCAATCTGTTGCAG & 60 \\
\hline 561 & TCTCCTTCAAGCATTCTGTCGACCCTGGATGTTGAATTGC & 0 \\
\hline 601 & 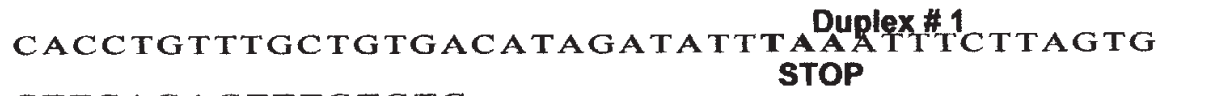 & \\
\hline 641 & СTTCAGAGTTTGTGTG & \\
\hline
\end{tabular}

Figure 1. A design of PTTG small interfering RNAs (siRNAs). Locations of PTTG siRNA nos. 1, 2, 3 and 4 examined in this study are shown by bold and underlined letters. Bold: ATG, translation initiation site; and TAA, translation termination site.

calculated by the following formula: $\mathrm{V}=(\text { width })^{2} \mathrm{x}$ length/2 (31). Two weeks after injection of cells, when small tumors had developed, animals were injected with $50 \mu 1$ of PBS containing control siRNA or PTTG siRNA no. 3 duplex to a final concentration of $50 \mu \mathrm{M}$ at the tumor site (32). Controls were injected with PBS only. The animal experiments were conducted in compliance with the University of Louisville Institutional Animal Use and Care Committee. The animals were sacrificed four weeks after tumor cell injection. Tumors were harvested and weighed, and divided into two parts, one part was fixed in $10 \%$ buffered formalin, and the remainder was stored in liquid nitrogen for later analysis.

Immunohistochemical analysis. For immunohistochemical analysis, 5- $\mu \mathrm{m}$ sections were cut from paraffin-embedded tissue, and mounted on slides. Slides were deparaffinized in xylene, hydrated with alcohol and stained with H\&E. For analysis of expression of PTTG, bFGF, Ki67 and CD34 proteins, the slides were placed in $3 \% \mathrm{H}_{2} \mathrm{O}_{2}$ /methanol solution for $20 \mathrm{~min}$ to quench endogenous peroxidase activity. The sections were incubated at $95^{\circ} \mathrm{C}$ in $0.01 \mathrm{~mol} / 1$ sodium citrate buffer ( $\mathrm{pH}$ 6.0) for $10 \mathrm{~min}$ to optimize antigen retrieval. Sections were pretreated with $5 \%$ normal goat serum for $60 \mathrm{~min}$ to block non-specific binding sites and were incubated overnight with PTTG antiserum diluted 1:1,500, Ki67 polyclonal antibody diluted 1:100, bFGF monoclonal antibody diluted $1: 100$ or CD34 diluted $1: 100$ at $4^{\circ} \mathrm{C}$. Control sections were incubated with preimmune serum, rabbit normal $\mathrm{IgG}$ or mouse IgG. Sections were washed three times (5 min each) with PBS and incubated at room temperature with biotinylated anti-rabbit secondary antibody for $30 \mathrm{~min}$ at room temperature. The sections were rinsed with PBS buffer and incubated with streptavidin-horseradish peroxidase-conjugated anti-rabbit IgG (Vector Laboratories, Burlingame, CA). After washing three times with PBS, immunoreactivity was visualized by incubating with diaminobenzidine tetrahydrochloride at room temperature until the desired intensity developed. The slides were rinsed with tap water and counterstained with hematoxylin (32). All slides were analyzed by two independent persons.

\section{Results}

PTTG is highly expressed in lung tumors and lung tumor cell lines. Northern blot analysis of the lung tumor tissues from squamous cell carcinoma, poorly differentiated large cell carcinoma, moderately differentiated squamous cell carcinoma and non-small cell carcinoma showed high levels of expression of PTTG mRNA compared to normal lung tissue (Fig. 2). High levels of expression of PTTG were also observed in lung tumor derived cell lines A549, lung carcinoma and H1299, non-small cell lung carcinoma cell lines. Consistent with these results, immunohistochemical analysis of the lung tumor tissues using the PTTG-specific antiserum showed high levels of PTTG protein compared to normal lung (Fig. 3). Expression of PTTG protein appears to be specific in lung tumor tissues, since no staining, or only a low level of staining was observed when preimmune serum was used.

To confirm the expression of PTTG in lung tumor tissues, we used a lung cancer profiling array (Clontech) that included normalized cDNA from 30 tumor and 30 corresponding normal tissues from individual patients, along with negative and positive controls, and cDNA from three cancer cell lines. A high level of PTTG expression was observed in 27 out of 30 tumor tissues. In normal tissue, expression of PTTG was 
A

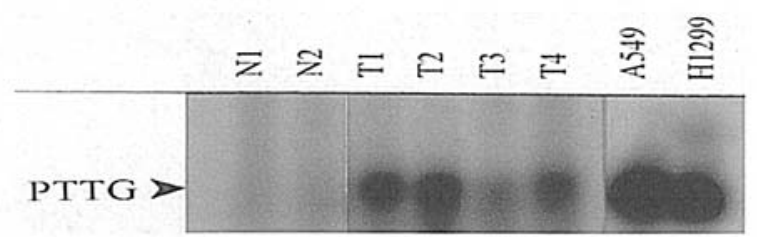

B

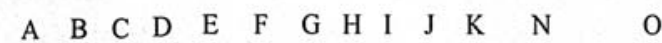

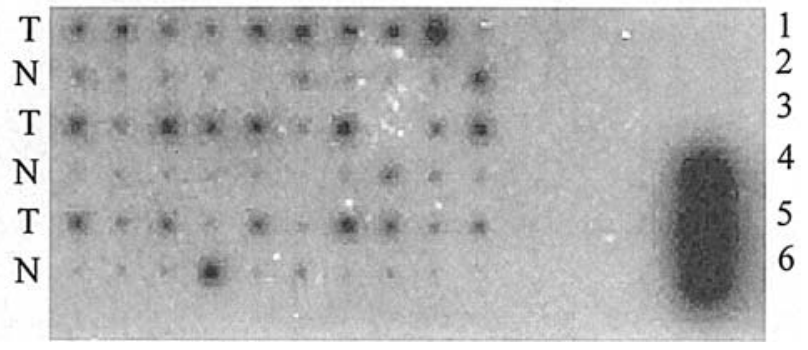

Figure 2. Expression of PTTG mRNA in lung tumor and normal tissues. A, Northern blot analysis of total RNA prepared from lung tumor and normal tissues. Each lane contains $20 \mu \mathrm{g}$ of total RNA. The blot was hybridized with [ $\left.{ }^{32} \mathrm{P}\right]$-labeled full length PTTG cDNA. N1 and N2, normal lung tissue; $\mathrm{T} 1$, squamous cell carcinoma of lung; T2, poorly differentiated large cell carcinoma of lung; T3, moderately differentiated large squamous cell carcinoma of lung; T4, non-small cell carcinoma of lung; A549 and H1299, lung tumor cell lines. B, sample lung cancer profiling array. Lung cancer profiling array was hybridized with $\left[{ }^{32} \mathrm{P}\right]$-labeled securin cDNA. N, normal; T, tumor. found to be very low or undetectable except in three tissue samples (Fig. 2). All three cell lines (A549, NCI-H460, and H1299) showed high levels of expression of PTTG (Fig. 2). No hybridization signal was detected in negative controls (yeast total RNA, yeast tRNA, poly (A) RNA, human $\mathrm{C}_{0} \mathrm{t}-$ DNA, human genomic DNA and ubiquitin cDNA), indicating the specificity of hybridization. Collectively, our results clearly demonstrate overexpression of PTTG in most of the lung tumors and tumor-derived cell lines.

Transfection of lung tumor cells with PTTG siRNA downregulates expression of PTTG $m R N A$ and protein. To test the efficacy of PTTG siRNA in down-regulation of expression of PTTG, we initially used a smart pool of four siRNA sequences derived from various regions of the PTTG coding sequence (pool of duplex no. 1, duplex no. 2, duplex no. 3 and duplex no. 4) (Fig. 1). Transfection of H1299 lung tumor cells with smart pool siRNA reduced PTTG mRNA levels $\sim 50 \%$ in $48 \mathrm{~h}$ as compared to untransfected cells or cells transfected with control siRNA (results not shown). Next, we tested the individual duplex siRNAs to determine the effect on downregulation of PTTG mRNA. Northern blot analysis showed down-regulation of PTTG by duplex no. 1 , no. 2 and no. 3 but not by duplex no. 4 within $24 \mathrm{~h}$ of transfection. Based on these results, we selected duplex no. 3 (5'-UGG GAG AUC UCA AGU UUC A-3'), which showed the best results as the most highly functional PTTG siRNA. Transfection of H1299

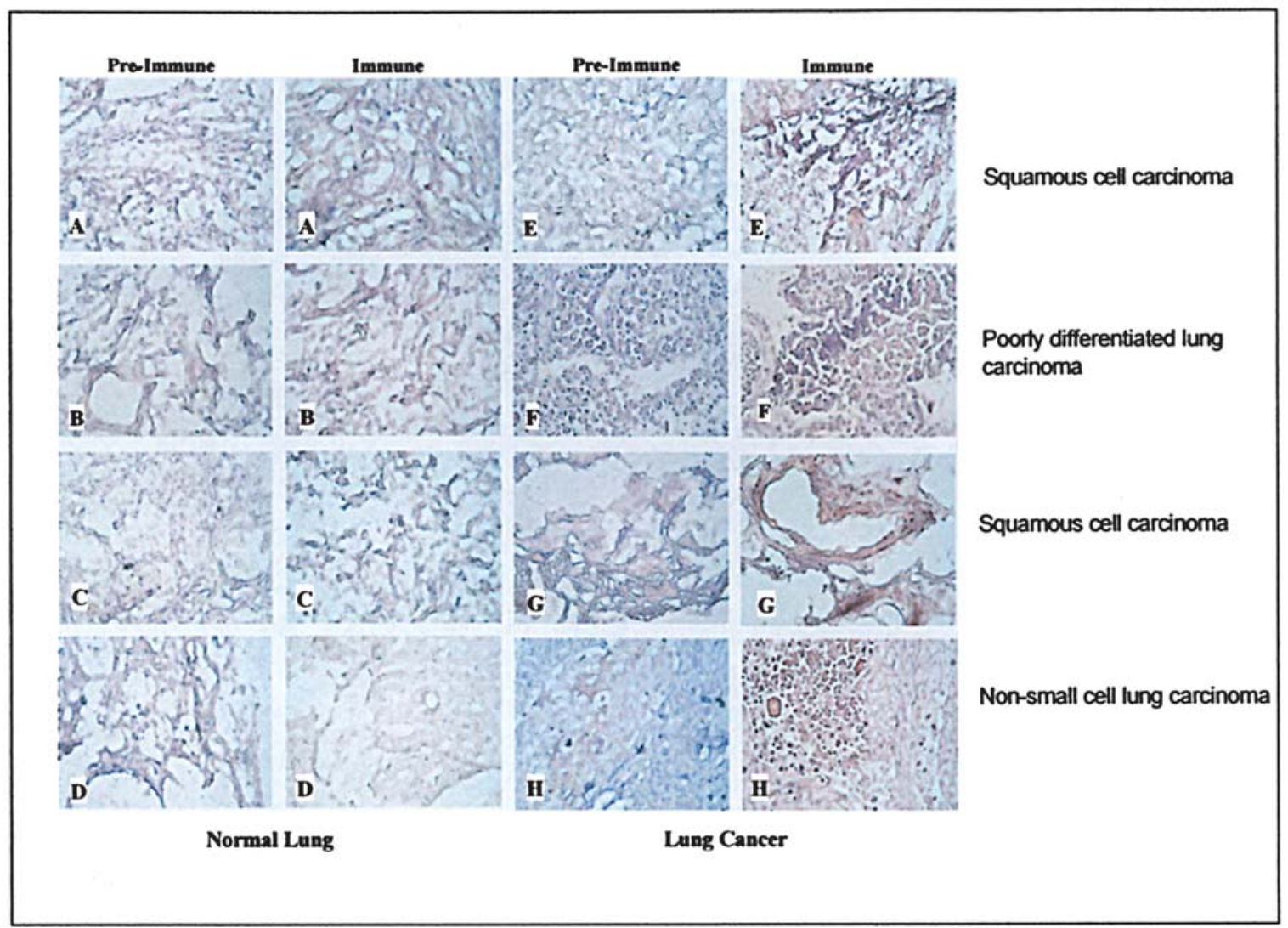

Figure 3. Immunohistochemical analysis of human normal and lung cancer tissues for expression of PTTG protein. PTTG protein-specific antiserum was used for analysis. Preimmune and immune serum was diluted at 1:1,500. A-D, normal lung tissue; E-H, lung cancer tissues; E, squamous cell carcinoma; F, poorly differentiated lung carcinoma; $\mathrm{G}$, squamous cell carcinoma; H, non-small cell carcinoma. The sections were counterstained with hematoxylin. Tumor cells (stained) surrounded by normal cells (unstained) in the same histological sections are shown (E-H/immune). 


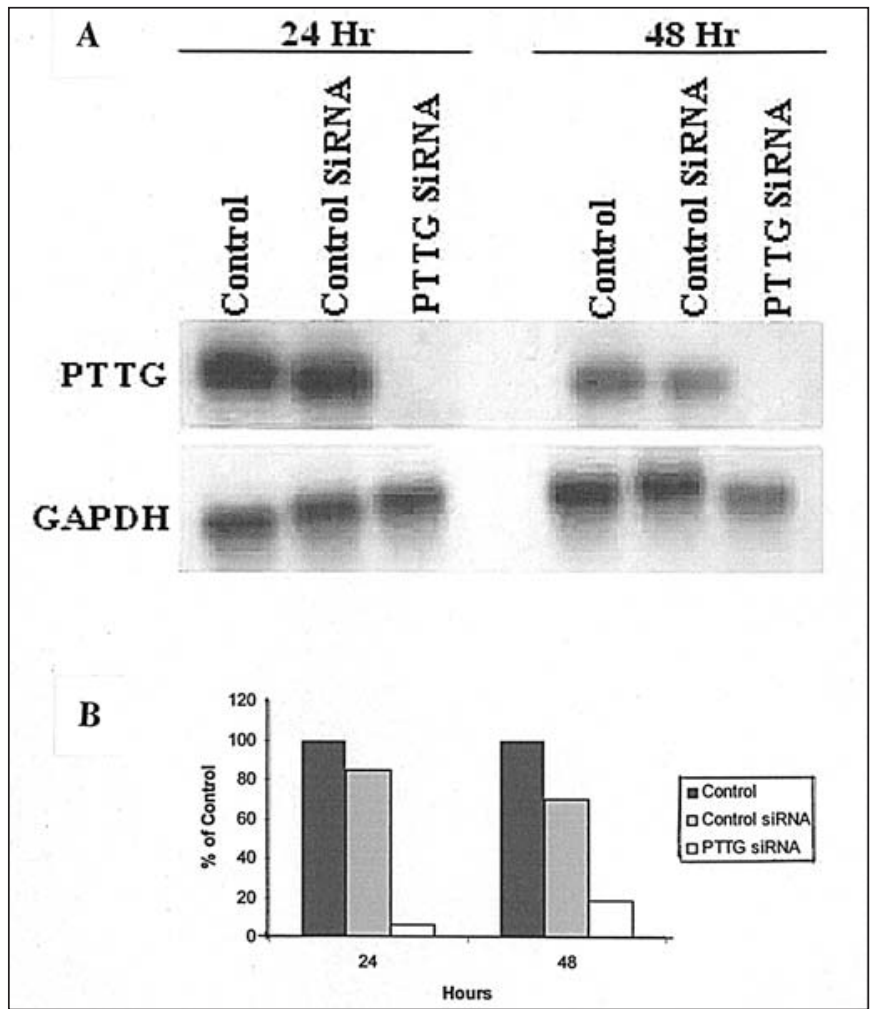

Figure 4. Effect of PTTG siRNA on expression of PTTG mRNA. A, lung tumor cells H1299 were transfected with PTTG siRNA or control siRNA for 24 or $48 \mathrm{~h}$. Total RNA was prepared and subjected to Northern blot analysis. $\left[{ }^{32} \mathrm{P}\right]$-labeled PTTG cDNA was used as a probe. GAPDH was used as an internal control. B, graphical representation of data shown in A. Data shown represent mean of two independent experiments.

cells with PTTG siRNA no. 3 to a final concentration of $100 \mathrm{nmol} / 1$, resulted in almost complete depletion of PTTG mRNA within 24-48 h of transfection compared to untransfected cells or cells transfected with control siRNA (Fig. 4). This siRNA duplex did not cause non-specific suppression of mRNA levels as shown by expression of GAPDH mRNA which was unchanged after transfection with the same siRNA. Western blot analysis of H1299 cells transfected with PTTG siRNA no. 3 showed $\sim 60 \%$ reduction in PTTG protein within $48 \mathrm{~h}$ of transfection (Fig. 5). Again suppression of PTTG protein levels by PTTG siRNA duplex appear to be specific since no change in $\beta$-actin was observed. Collectively, these results indicate that PTTG siRNA is capable of downregulating the expression of PTTG mRNA and protein.

Knockdown of PTTG inhibits colony formation. In phenotype analysis, we investigated the effect of PTTG siRNA duplex no. 3 on colony formation of H1299 cells. The siRNA transfection was performed for $48 \mathrm{~h}$ at $100 \mathrm{nmol} / \mathrm{l}$ to achieve complete suppression of PTTG mRNA expression. For colony formation assay, $10^{4}$ cells after transfection with siRNA were plated in soft agar as described previously (5). As shown in Fig. 6, the number of colonies formed by H12999 cells transfected with PTTG siRNA was significantly reduced as compared to untransfected cells or cells transfected with control siRNA. These results indicate that the siRNA-mediated specific knockdown of PTTG induced strong inhibition of lung tumor cell growth in vitro.

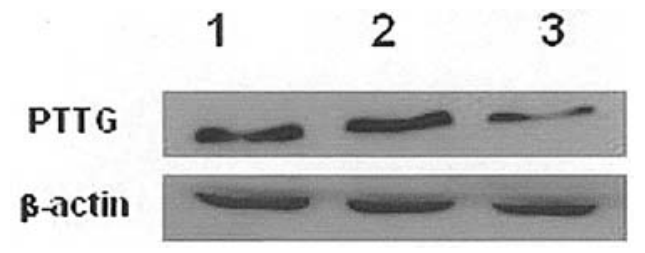

Figure 5. Western blot analysis of H1299 cells transfected with PTTG siRNA or control siRNA. After $48 \mathrm{~h}$ of transfection, cells were harvested and subjected to Western blot analysis. $\beta$-actin was used as a control. Lane 1, untransfected cells; lane 2, cells transfected with control siRNA; and lane 3, cells transfected with PTTG siRNA. Data are representative of two independent experiments.

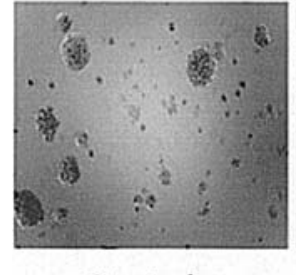

Control

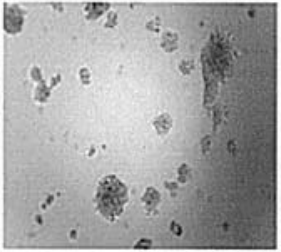

Control siRNA

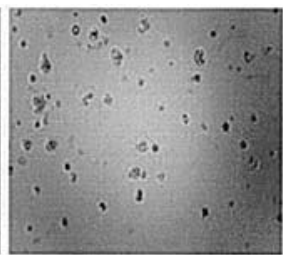

PTTG-siRNA
Figure 6. Effect of PTTG siRNA on anchorage-independent growth (colony formation on soft agar) of lung tumor cells. H1299 cells were transfected with PTTG siRNA or control siRNA. After $48 \mathrm{~h}$ of transfection, cells were harvested and plated on soft agar. Colony formation was examined after 14 days of plating and photographed. Data are representative of three independent experiments.

Knockdown of PTTG suppresses tumor development in nude mice. To determine whether suppression of PTTG expression also suppresses lung tumor growth in vivo, H1299 cells were transfected with PTTG siRNA to a final concentration of $100 \mathrm{nmol} / 1$, and injected into nude mice. As expected, untransfected H1299 cells and cells transfected with control siRNA produced visible tumors at the injection sites within two weeks of injection, whereas cells transfected with PTTG siRNA no. 3 failed to develop tumors during this time. Beyond two weeks of injection, animals injected with cells transfected with PTTG siRNA duplex no. 3 also developed tumors. However, three out of five animals developed tumors and tumor growth in these animals was significantly less than control untransfected cells or cells transfected with control siRNA (Fig. 7). Tumor mass at the end of the experiment (four weeks after injection of cells) was significantly lower in animals injected with $\mathrm{H} 1299$ cells transfected with PTTG siRNA duplex no. $3(67.85 \pm 45.87 \mathrm{mg})$ compared to untransfected cells $(232.12 \pm 102.78 \mathrm{mg})$ or cells transfected with control siRNA $(231.57 \pm 83.76 \mathrm{mg})$. No gross adverse affects in animals, such as loss of appetite or body weight were observed during the experimental periods. These results indicate that siRNA-mediated knockdown of PTTG exerted a strong antitumorigenic effect in vivo.

Expression of PTTG, Ki67, bFGF and CD34 in tumors. To assess the relationship between the antitumor effects of PTTG siRNA and tumor associated cell growth and neovascularization, we stained tumor tissues with PTTG, Ki67, bFGF and CD34 using specific antibodies for each protein. 


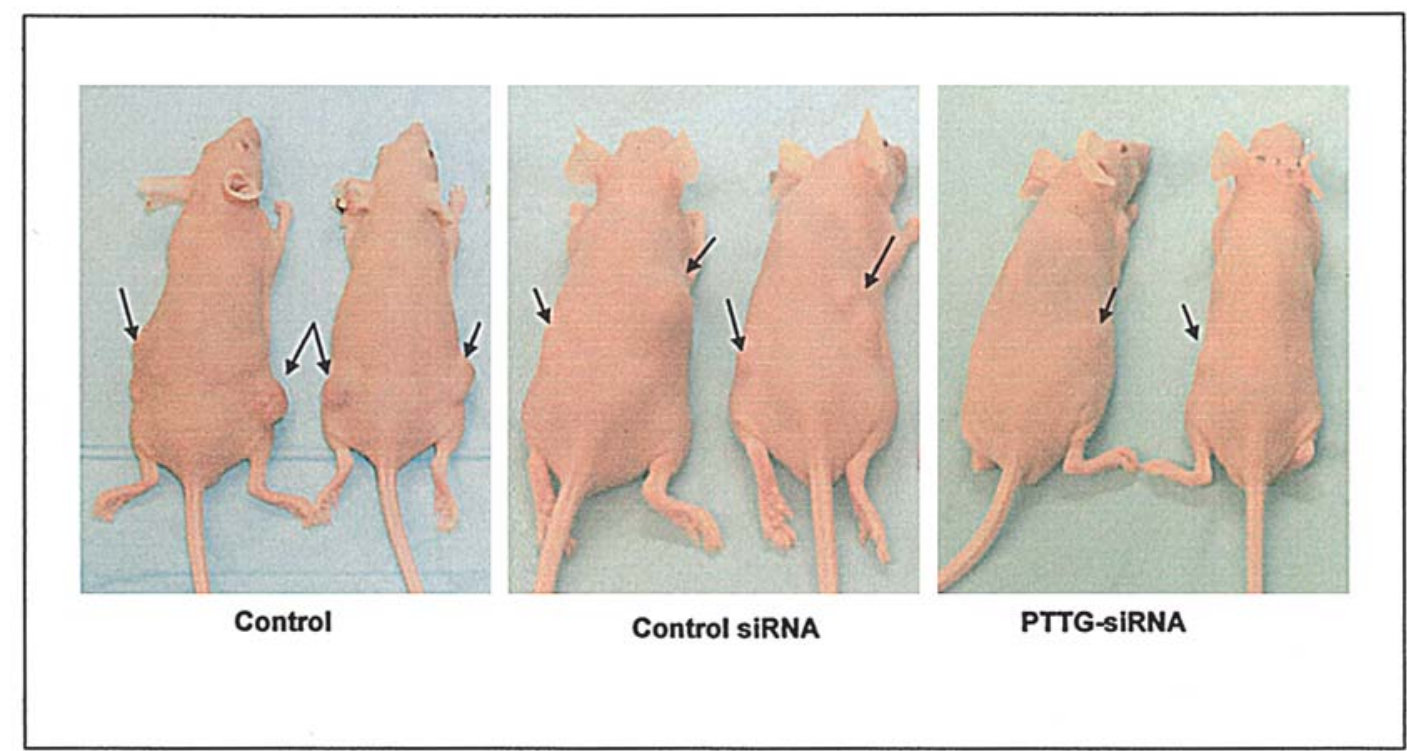

Figure 7. Effect of PTTG siRNA on tumor development in nude mice. H1299 cells were transfected with PTTG siRNA or control siRNA and injected s.c. into nude mice. After 14 days of injection, siRNA or control siRNA was injected into the tumor site. Controls were injected with PBS. Arrows indicate the tumors. Tumors that developed after 4 weeks of injection of cells were measured, excised and weighed.

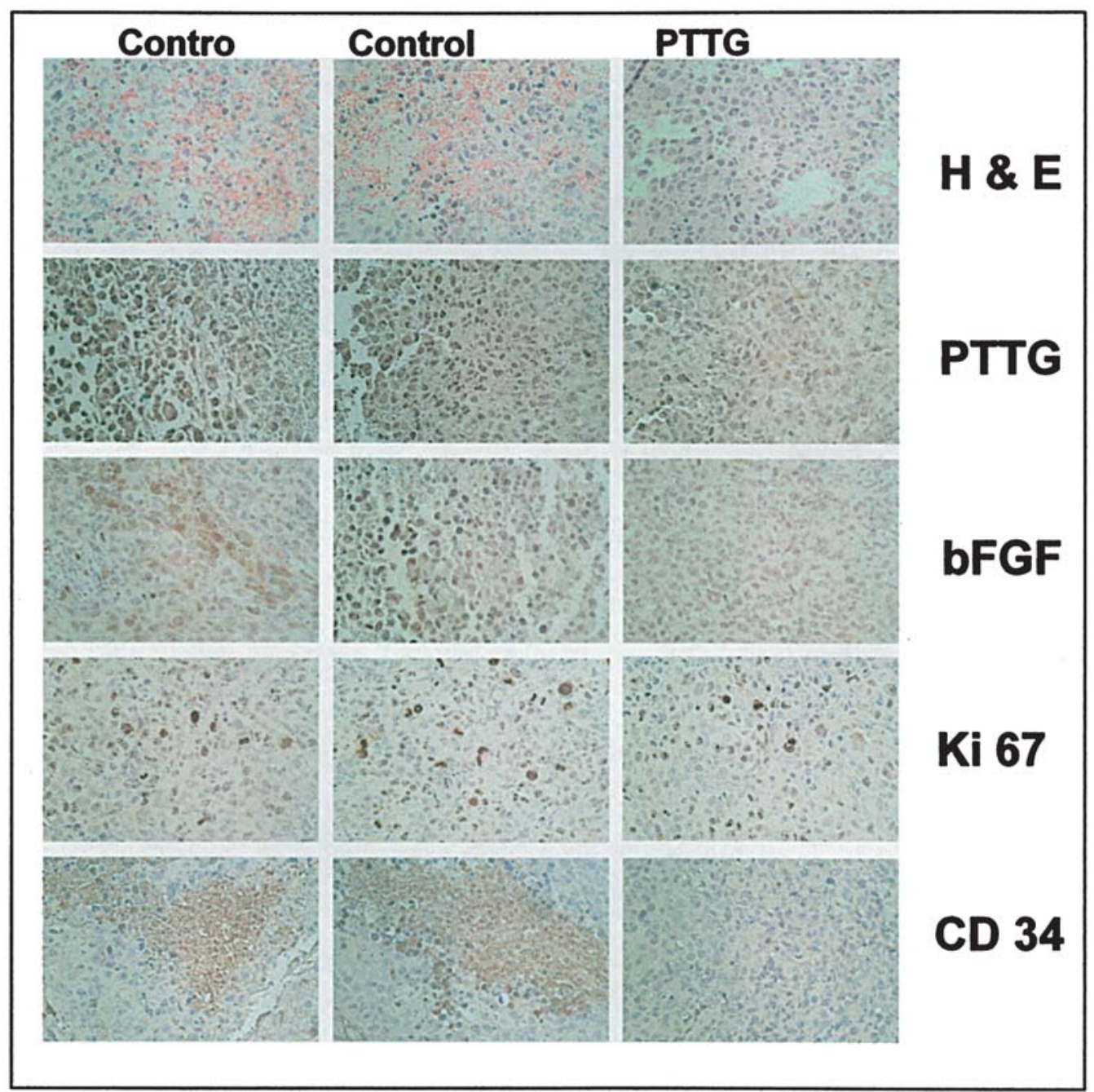

Figure 8. Immunohistochemical analysis of the expression of PTTG, bFGF, Ki67 and CD34 in tumors that developed in nude mice injected with untransfected H1299 cells, cells transfected with control siRNA or PTTG siRNA. Specific antibody for each protein was used. Multiple sections for each tumor were examined under x70 Olympus microscope by two independent investigators. Data shown is representative of three independent experiments and multiple sections for each tissue. 
Representative results are shown in Fig. 8. H\&E staining of tumors developed from untransfected cells or cells transfected with control siRNA showed necrosis, which was absent or reduced in tumors developed from cells transfected with PTTG siRNA. PTTG, bFGF and CD34 were significantly reduced in tumors developed from H1299 cells transfected with PTTG siRNA duplex no. 3 followed by treatment with PTTG siRNA compared to untransfected cells or cells transfected with control siRNA. Similarly, expression of Ki 67 was significantly reduced suggesting a reduction in cell proliferation.

\section{Discussion}

In this study using Northern blot analysis and immunohistochemical techniques, we show that PTTG is highly expressed in many lung tumor tissues compared to normal lung (Figs. 2 and 3). PTTG is a commonly overexpressed gene in various types of cancer $(5,6,9-18)$ and is believed to play an important role in augmenting tumor angiogenesis, metastasis and growth. Overexpression of PTTG has been shown to induce cellular transformation and to promote tumor development in nude mice. Therefore, specific down-regulation of PTTG might be a potential treatment strategy against human cancers, including lung cancer. In fact, suppression of PTTG expression by fulllength antisense mRNA (33), or oligonucleotides against PTTG mRNA (34) were previously reported to inhibit proliferation of cancer cell lines in vitro. However, these techniques have been difficult to apply universally (35). The advent of RNAi directed 'knock-down' has sparked a revolution in somatic cell genetics, allowing an inexpensive and rapid analysis of gene function in mammals, and represents an approach that might be exploited for gene therapy (36). Some studies directly compared RNAi with antisense RNA and found RNAi to be quantitatively more efficient and durable in cell culture and in nude mice (37).

In order to explore PTTG as a therapeutic target, we employed the siRNA technique for knockdown of its expression and analyzed the phenotype. Our study using lung tumor H1299 cells shows that siRNA targeting human PTTG can be efficiently delivered to lung cancers, and rapidly inhibits the expression and activity of PTTG. Inhibition of PTTG activity is associated with reduced colony formation on soft agar and suppression of tumor development in nude mice. Our study showed $\sim 80 \%$ reduction in PTTG mRNA and $60 \%$ reduction in PTTG protein within $48 \mathrm{~h}$ of transfection of cells with siRNA (Figs. 4 and 5). Consistent with our results, Chesnokova et al (38) recently showed a significant reduction in tumor development in PTTG (-/-)/Rb (+/-) animals, supporting the hypothesis that depletion of PTTG in cancers can reverse the cancer phenotype. However, the mechanisms by which suppression of PTTG expression results in inhibition of tumor growth and development remain unclear.

Securins are regulatory proteins that are associated with a cysteine protease called separase, play an important role in sister chromatid separation during $\mathbf{M}$ phase (39), and exhibit characteristics of cell cycle regulators. Depletion of securin (cut2) in fission yeast and PIM in Drosophila has been reported to be lethal $(40,41)$. However, deletion of securin in budding yeast $(\mathrm{Pds} 1 \mathrm{P})$ and vertebrate (PTTG) genes results in increased rates of chromosome loss but not necessarily lethality $(42,43)$. Mice lacking PTTG are viable and fertile (44), but showed diminished $\beta$ cell mass, with impaired glucose homeostasis leading to diabetes during late adulthood (45), suggesting a defect in $B$ cell division.

In this study, we showed that suppression of PTTG expression is associated with reduction of expression of Ki67 and bFGF. Ki67 expression is a cell proliferation marker $(46,47)$. Reduced expression of PTTG correlates with the expression of Ki67, suggesting a relationship between PTTG and cell proliferation. Similarly, Chesnokova et al (38) showed reduced cell proliferation and Ki67 expression in pituitary cells derived from Rb (+/-)/PTTG (-/-) animals compared to wild-type animals, suggesting reduction of pituitary cell proliferation if PTTG is absent. Basic fibroblast growth factor (bFGF) is known to play an important role in tumor angiogenesis and metastasis $(48,49)$ and is up-regulated by PTTG (20). In previous studies, we showed that the secretion and expression of bFGF in HEK293 cells are increased following transfection with PTTG cDNA and in tumors developed on injection of HEK293 cells expressing PTTG (19), suggesting that the decrease in expression of bFGF when expression of PTTG was reduced may represent one of the mechanisms by which PTTG achieves its antitumor function.

Inhibition of PTTG activity was also associated with a remarkable decrease in CD34-positive cells in tumors that developed in nude mice from cells transfected with PTTG siRNA duplex no. 3 (Fig. 8). CD34 is thought to play an important role in the development of new vessels, tumor progression and the recruitment of stem cells into tumors (50). Reduction of CD34-positive cells as a result of reduction of expression of PTTG suggests a novel role and mechanism for PTTG in tumorigenesis. It is possible that overexpression of PTTG in normal cells increases recruitment of progenitor cells (stem cells) that help in tumor growth and progression.

In conclusion, we demonstrated that decreasing PTTG expression by the use of PTTG siRNA inhibits tumor growth both in vitro and in vivo. Further study is needed to test whether PTTG expression can be efficiently depleted by siRNA expressed from a DNA-based expression vector combined with a tumor-specific promoter, such that siRNA can specifically target PTTG in cancer cells without affecting normal cells.

\section{Acknowledgments}

The authors are thankful to Dr Stephen J. Winters for his help in editing the manuscript and for his valuable comments. This work was supported by a grant from the Kentucky Lung Cancer Program.

\section{References}

1. Jemal A, Murray T, Ward E, Samuels A, Tiwari RC, Ghafoor A, Feuer EJ and Thun MJ: Cancer statistics. CA Cancer J Clin 55: 10-30, 2005.

2. Mulligan-Kehoe MJ and Ross A: General concepts of molecular biology related to lung cancer. In: Lung Cancer. Principal and Practice. 2nd edition. Pass H, Mitchell J, Johnson D, Turrisi A and Minna J (eds). Lippincott Williams and Wilkins, Philadelphia, pp5-66, 2000.

3. Travis WD, Linder J and Mackay B: Classification, histology, cytology and electron microscopy. In: Lung Cancer. Principal and Practice. 2nd edition. Pass H, Mitchell J, Johnson D, Turrisi A and Minna J (eds). Lippincott Williams and Wilkins, Philadelphia, pp435-495, 2000. 
4. Pei L and Melmed S: Isolation and characterization of a pituitary tumor-transforming gene (PTTG). Mol Endocrinol 11: 433-441, 1997.

5. Kakar SS and Jennes L: Molecular cloning and characterization of the tumor transforming gene (TUTR1): a novel gene in human tumorigenesis. Cytogenet Cell Genet 84: 211-216, 1999.

6. Puri R, Tousson A, Chen L and Kakar SS: Molecular cloning of pituitary tumor transforming gene 1 from ovarian tumors and its expression in tumors. Cancer Lett 163: 131-139, 2001.

7. Kakar SS: Assignment of the human tumor transforming gene TUTR1 to chromosome band $5 q 35.1$ by fluorescence in situ hybridization. Cytogenet Cell Genet 83: 93-95, 1998.

8. Hosoe S, Ueno K, Shigedo Y, Tachibana I, Osaki T, Kumagai T, Tanio Y, Kawase I, Nakamura Y and Kishimoto T: A frequent deletion of chromosome 5q21 in advanced small cell and nonsmall cell carcinoma of the lung. Cancer Res 54: 1787-1790, 1994.

9. Ramos-Morales F, Dominguez A, Romero F, Luna R, Multon MC, Pintor-Toro JA and Tortolero M: Cell cycle regulated expression and phosphorylation of hpttg proto-oncogene product. Oncogene 19: 403-409, 2000.

10. Dominguez A, Ramos-Morales F, Romero F, Rios RM, Dreyfus F, Tortolero M and Pintor-Toro JA: hpttg, a human homologue of rat pttg, is overexpressed in hematopoietic neoplasms. Evidence for a transcriptional activation function of hPTTG. Oncogene 17: 2187-2193, 2000.

11. $\mathrm{Yu} \mathrm{R}$ and Melmed S: Oncogene activation in pituitary tumors. Brain Pathol 11: 328-341, 2001.

12. Heaney AP, Singson R, McCabe CJ, Nelson V, Nakashima M and Melmed S: Expression of pituitary-tumour transforming gene in colorectal tumours. Lancet 355: 716-719, 2000.

13. Kakar SS, Chen L, Puri R, Flynn SE and Jennes L: Characterization of a polyclonal antibody to human pituitary tumor transforming gene 1 (PTTG1) protein. J Histochem Cytochem 49: 1537-1546, 2001.

14. Heaney AP, Fernando M and Melmed S: Functional role of estrogen in pituitary tumor pathogenesis. J Clin Invest 109: 277-283, 2002

15. Boelaert K, McCabe CJ, Tannahill LA, Gittoes NJ, Holder RL, Watkinson JC, Bradwell AR, Sheppard MC and Franklyn JA: Pituitary tumor transforming gene and fibroblast growth factor-2 expression: potential prognostic indicators in differentiated thyroid cancer. J Clin Endocrinol Metab 88: 2341-2347, 2003.

16. Saez C, Japon MA, Ramos-Morales F, Romero F, Segura DI, Tortolero $\mathbf{M}$ and Pintor-Toro JA: hpttg is over-expressed in pituitary adenomas and other primary epithelial neoplasias. Oncogene 18: 5473-5476, 1999.

17. Ramaswamy S, Ross KN, Lander ES and Golub TR: A molecular signature of metastasis in primary solid tumors. Nat Genet 33: 49-54, 2003.

18. Shibata Y, Haruki N, Kuwabara Y, Nishiwaki T, Kato J, Shinoda N, Sato A, Kimura M, Koyama H, Toyama T, Ishiguro H, Kudo J, Terashita Y, Konishi S and Fujii Y: Expression of PTTG (pituitary tumor transforming gene) in esophageal cancer. Jap J Clin Oncol 32: 233-237, 2002.

19. Hamid T, Malik MT and Kakar SS: Ectopic expression of PTTG1/securin promotes tumorigenesis in human embryonic kidney cells. Mol Cancer 13: 1-13, 2005.

20. Ishikawa H, Heaney AP, Yu R, Horwitz GA and Melmed S: Human pituitary tumor-transforming gene induces angiogenesis. J Clin Endocrinol Metab 86: 867-874, 2001.

21. Heaney AP, Horwitz GA, Wang Z, Singson R and Melmed S: Early involvement of estrogen-induced pituitary tumor transforming gene and fibroblast growth factor expression in prolactinoma pathogenesis. Nat Med 5: 1317-1321, 1999.

22. McCabe CJ, Boelaert K, Tannahill LA, Heaney AP, Stratford AL, Khaira JS, Hussain S, Sheppard MC, Franklyn JA and Gittoes NJ: Vascular endothelial growth factor, its receptor KDR/Flk-1, and pituitary tumor transforming gene in pituitary tumors. J Clin Endocrinol Metab 87: 4238-4244, 2002.

23. Fire A, Xu S, Montgomery MK, Kostas SA, Driver SE and Mello CC: Potent and specific genetic interference by doublestranded RNA in Caenorhabditis elegans. Nature 391: 806-811, 1998.

24. Hammond SM, Bernstein E, Beach D and Hannon GJ: An RNA-directed nuclease mediates post-transcriptional gene silencing in Drosophila cells. Nature 404: 293-296, 2000.

25. Bernstein E, Denli AM and Hannon GJ: The rest is silence. RNA-A Publ RNA Soc 7: 1509-1521, 2001.
26. Hammond SM, Caudy AA and Hannon GJ: Post-transcriptional gene silencing by double-stranded RNA. Nat Rev Genet 2: 110-119, 2001.

27. Reynolds A, Leake D, Boese Q, Scaringe S, Marshall WS and Khvorova A: Rational siRNA design for RNA interference. Evaluation Studies. Nat Biotech 22: 326-330, 2004.

28. Kaul D and Sikand K: Defective RNA-mediated c-myc gene silencing pathway in Burkitt's lymphoma. Biochem Biophys Res Commun 313: 552-554, 2004.

29. Yoshinouchi M, Yamada T, Kizaki M, Fen J, Koseki T, Ikeda Y, Nishihara $\mathrm{T}$ and Yamato $\mathrm{K}$ : In vitro and in vivo growth suppression of human papillomavirus 16-positive cervical cancer cells by E6 siRNA. Mol Ther 8: 762-768, 2003.

30. Futami T, Miyagishi M, Seki M and Taira K: Induction of apoptosis in HeLa cells with siRNA expression vector targeted against bcl-2. Nucleic Acids Res 2 (suppl): 251-252, 2002.

31. Burfeind P, Chernicky CL, Rininsland F, Ilan J and Ilan J: Antisense RNA to the type I insulin-like growth factor receptor suppresses tumor growth and prevents invasion by rat prostate cancer cells in vivo. Proc Natl Acad Sci USA 93: 7263-7268, 1996.

32. Takei Y, Kadomatsu K, Yuzawa Y, Matsuo S and Muramatsu T: A small interfering RNA targeting vascular endothelial growth factor as cancer therapeutics. Cancer Res 64: 3365-3370, 2004.

33. Chen G, Li J, Li F, Li X, Zhou J, Lu Y and Ma D: Inhibitory effects of anti-sense PTTG on malignant phenotype of human ovarian carcinoma cell line SK-OV-3. J Huazhong Univ Sci Technolog Med Sci 24: 369-372, 2004.

34. Solbach C, Roller M, Peters S, Nicoletti M, Kaufmann M and Knecht R: Pituitary tumor-transforming gene (PTTG): a novel target for anti-tumor therapy. Anticancer Res 25: 121-125, 2005.

35. Braasch DA, Liu Y and Corey DR: Antisense inhibition of gene expression in cells by oligonucleotides incorporating locked nucleic acids: effect of mRNA target sequence and chimera design. Nucleic Acids Res 30: 5160-5167, 2002.

36. Hannon GJ: RNA interference. Nature 418: 244-251, 2002.

37. Bertrand JR, Pottier M, Vekris A, Opolon P, Maksimenko A and Malvy C: Comparison of antisense oligonucleotides and siRNAs in cell culture and in vivo. Biochem Biophys Res Commun 296: 1000-1004, 2002.

38. Chesnokova V, Kovacs K, Castro AV, Zonis S and Melmed S: Pituitary hypoplasia in $\mathrm{Pttg}^{-/-}$mice is protective for $\mathrm{Rb}^{+/}$ pituitary tumorigenesis. Mol Endocrinol 19: 2371-2379, 2005.

39. Nasmyth K, Peters JM and Uhlmann F: Splitting the chromosome: cutting the ties that bind sister chromatids. Science 288 : 1379-1385, 2000.

40. Ciosk R, Zachariae W, Michaelis C, Shevchenko A, Mann M and Nasmyth K: An ESP1/PDS1 complex regulates loss of sister chromatid cohesion at the metaphase to anaphase transition in yeast. Cell 93: 1067-1076, 1998.

41. Stratmann R and Lehner CF: Separation of sister chromatids in mitosis requires the Drosophila pimples product, a protein degraded after the metaphase/anaphase transition. Cell 84: 25-35, 1996

42. Jallepalli PV, Waizenegger IC, Bunz F, Langer S, Speicher MR, Peters JM, Kinzler KW, Vogelstein B and Lengauer C: Securin is required for chromosomal stability in human cells. Cell 105: 445-457, 2001.

43. Yamamoto A, Guacci V and Koshland D: Pds1p, an inhibitor of anaphase in budding yeast, plays a critical role in the APC and checkpoint pathway(s). J Cell Biol 133: 99-110, 1996.

44. Wang Z, Yu R and Melmed S: Mice lacking pituitary tumor transforming gene show testicular and splenic hypoplasia, thymic hyperplasia, thrombocytopenia, aberrant cell cycle progression, and premature centromere division. Mol Endocrinol 15: 1870-1879, 2001.

45. Wang Z, Moro E, Kovacs K, Yu R and Melmed S: Pituitary tumor transforming gene-null male mice exhibit impaired pancreatic beta cell proliferation and diabetes. Proc Natl Acad Sci USA 100: 3428-3432, 2003.

46. Gerdes J, Schwab U, Lemke H and Stein H: Production of a mouse monoclonal antibody reactive with a human nuclear antigen associated with cell proliferation. Int J Cancer 31: 13-20, 1983 .

47. Gerdes J, Lemke H, Baisch H, Wacker HH, Schwab U and Stein H: Cell cycle analysis of a cell proliferation-associated human nuclear antigen defined by the monoclonal antibody Ki-67. J Immunol 133: 1710-1715, 1984. 
48. Fujimoto J, Ichigo S, Hori M, Hirose R, Sakaguchi $\mathrm{H}$ and Tamaya T: Expression of basic fibroblast growth factor and its mRNA in advanced ovarian cancers. Eur J Gynaecol Oncol 18: 349-352, 1997.

49. Di Blasio AM, Cremonesi L, Vigano P, Ferrari M, Gospodarowicz D, Vignali M and Jaffe RB: Basic fibroblast growth factor and its receptor messenger ribonucleic acids are expressed in human ovarian epithelial neoplasms. Am J Obstet Gynaecol 169: 1517-1523, 1993.

50. El-Shahat M, Lotfy M, Fahmy L, Abouel-Nour MF and ElKenawy Ael-M: Prognostic value of microvessel density, matrix metalloproteinase-9 and p53 protein expression in esophageal cancer. Egypt Natl Cancer Inst 16: 224-230, 2004. 